\title{
AN ONTOLOGICAL ANALYSIS AND NATURAL LANGUAge Processing Of Figures of SPEECH
}

\author{
Christiana Panayiotou \\ Department of Communications and Internet Studies, \\ Technological University of Cyprus
}

\begin{abstract}
The purpose of the current paper is to present an ontological analysis to the identification of a particular type of prepositional figures of speech via the identification of inconsistencies in ontological concepts. Prepositional noun phrases are used widely in a multiplicity of domains to describe real world events and activities. However, one aspect that makes a prepositional noun phrase poetical is that the latter suggests a semantic relationship between concepts that does not exist in the real world. The current paper shows that a set of rules based on WordNet classes and an ontology representing human behaviour and properties, can be used to identify figures of speech due to the discrepancies in the semantic relations of the concepts involved. Based on this realization, the paper describes a method for determining poetic vs. non-poetic prepositional figures of speech, using WordNet class hierarchies. The paper also addresses the problem of inconsistency resulting from the assertion of figures of speech in ontological knowledge bases, identifying the problems involved in their representation. Finally, it discusses how a contextualized approach might help to resolve this problem.
\end{abstract}

\section{KEYWORDS}

Ontologies, NLP, Linguistic creativity

\section{INTRODUCTION}

Research in computational linguistic creativity has gained a renewed attention over the last decade and falls under the auspices of Artificial Intelligence, Natural Language Processing and Linguistics. Poetry, as a special form of creative writing makes intense use of identifiable linguistic tools such as figures of speech [1]. Poetry, is characterized as Art partly due to its aesthetic qualities which appeal to the human senses and due to its notional and semantic content. In [2] poetry is defined as the art form in which human language is used for its aesthetic qualities in addition to, or instead of, its notional and semantic content. Poetic writings frequently violate the syntactical, phonological and semantic rules of natural language text [3]. However, they also possess some distinct characteristics that help to identify poetic writings among other text and set the grounds upon which the automatic recognition of poetic phrases can be done. Our analysis is based on the realization that certain literary tools e.g. figures of speech [4] violate ontological relationships among concepts in order to cause emotional and cognitive effects. Although we focused on a very simple subset of these phrases, our ideas can be expanded to more complex phrases in the future.

As a starting point to our work, a number of simple prepositional noun phrases were extracted from the Gutenberg files containing poems by William Blake and the Complete Works of Shakespeare [5]. Then, via the use of WordNet hypernym relations [6], conflict relations were identified giving rise to figures of speech [1]. Results were promising since even at this primitive stage we have been able to identify patterns leading to the identification of figures of speech. For 
example, let us consider the prepositional noun phrase: "the tent of God" appearing in the poem: "The Little Black Boy", by William Blake [8]. This prepositional noun phrase relates the words "tent" and "God" in the particular type of natural language phrase. The first three superordinates in the hypernym hierarchy of the word "tent" in WordNet [6], are: "shelter.n.01", "structure.n.01", and "artifact.n.01". The word "god" has the two immediate superordinates (e.g. the first two hypernyms in its hypernym hierarchy): "spiritual_being.n.01", and "belief.n.01". If we were to prototype the relevant phrases for clarity, we would arrive at phrases of the form:

\author{
the SHELTER of SPIRITUAL BEING \\ the ARTIFACT of SPIRITUAL_BEING
}

where in place of ARTIFACT we can place any noun word whose hypernym closure includes "artifact.n.01" e.g. the sense 0 interpretation of noun "tent" in WordNet, and similarly in the place of SPIRITUAL_BEING any noun word referring to a spiritual object whose hypernym closure includes "spiritual_being.n.01". Both of the above prototypes lead to semantic relations that are counter-intuitive when interpreted in the real world. The extraction of rules aiming to identify counter-intuitive relations between nouns, leading to the identification of figures of speech, is discussed in section 3 .

Our analysis also showed that there are no crisp boundaries in classification of textual entities and that the nouns associated in two phrases, one being poetic and another being non-poetic, may have common hypernyms in their hypernym closure. Also, although WordNet [6] is useful for relating words to concepts and to concept hierarchies, each synset [6] is connected to other synsets via the hypernym, hyponym and meronym semantic relations but not any other type of ontological domain-specific semantic relations. Any other domain-specific ontological relations need to be addressed via semantically enriched formalisms. Considering different senses [6] of synsets, is beyond the scope of the current work. The purpose of the next section is to introduce the notion of "figure of speech", to overview existing work in computational poetry and to refer to the fuzzy domain approaches towards classification and ontology generation. Section 3 provides a set of rules underpinning the recognition of figures of speech and provides a method for the derivation of figures of speech using WordNet hypernyms. It also provides a partially defined ontology of humanly possessed attributes whose hierarchical concepts can be mapped to hypernym classes and discusses the notion of consistency. Section 4 discusses practical considerations regarding consistency handling and representation.

\title{
2. BACKGROUND AND RELATED WORK
}

The main focus of research in computational creativity over the past decade has been in the creation of models capable of generating poetry and in the classification of poetry. Several models have been created for the automatic generation of poetry. However, none of these works to our awareness addresses the problem of automatic recognition of figure of speech [1] and the ontological analysis of poetic text. In this section, we discuss some of these approaches and we refer to the properties of poetic text that makes poetry generation and understanding a distinct challenging problem on its own.

Part of our work requires the mapping of WordNet entities to an ontology modelling Human attributes and behavior. Substantial research in the extraction of semantic relations from resources using fuzzy classification and similarity measures and the automatic generation of fuzzy ontologies has been cited in literature with applications in Information Retrieval [27, 28], eLearning [29] and Engineering [30]. The current work takes a semi-automatic iterative approach to the mapping of WordNet entities to an ontology whereby figures of speech are recognized as conflicting assertions giving rise to a set of rules used to extract figures of speech. The conflicting 
nature of assertions made by figures of speech has its own ontological significance into the understanding and analysis of processes that lead to poetic texts.

The structure of this section is outlined as follows: In the next subsection we define the meaning of the terms: figure of speech and personification, by reference to definitions provided by poetry resources. Then, we discuss some of the most important poetry generation approaches in literature, which has provided insight into the characteristics and complexities of poetic writings. Finally, we refer to important work in the fuzzy domain related to fuzzy classification, similarity measures and ontology generation.

\subsection{Figures of speech}

Before we discuss some of the most important works in this area, we need to explain the meanings of figure of speech [1] and personification [7], used extensively in this work. Knowledge of these terms, is essential to the understanding of the nature of the problem addressed, i.e. the ontological analysis of of the characteristics of figures of speech that make them distinct from other textual forms.

Definition 1 (Figure of Speech [1]) A figure of speech is a phrase or word having different meanings than its literal meaning. It conveys meaning by identifying or comparing one thing with another, which has connotation or meaning familiar to the audience. That is why it is helpful in creating a vivid rhetorical effect.

The above notion forms a particular type of poetry device used to cause aesthetic and cognitive events due to its paradoxical nature. A vivid example is the case of personification phrases constituting "a particular type of figures of speech where non-human objects are portrayed in such a way that we feel they have the ability to act like human beings" [7]. An example of personification appears in the poem: "Two Sunflowers move in a yellow room" by William Blake, whereby two sunflowers are assumed to be moving into a room and conversing with the poet, saying: "Our traveling habits have tired us. Can you give us a room with a view?". Another example can be traced to Emily Dickinson's poem: "Because I could not stop for dead" whereby Death is personified as shown in the following lines:

"Because I could not stop for Death.

He kindly stopped for me

The Carriage held but just Ourselves

And Immortality.

We slowly drove

He knew no haste. [...]"

In the above example, the lines: "he kindly stopped for me" and "he knew no haste" attribute human qualities to Death and are vivid examples of personification phrases.

Figures of speech appear frequently in Poetry. Identifying these phrases, and investigating the semantic relationships of the objects involved in them, sets the ground for further analysis of poetic text. Neither the extraction, nor the identification, of these phrases have been addressed before.

\subsection{Existing Work in Computational Poetry}

Among the first advocates of automatic generation of poetry is R.W. Bailey [9]. Toivanen et al. [10] introduced predicates which explicitly recorded the possible words that can exist at each line and each position in a poem combined with constraints e.g. that only one candidate word can 
exist in a particular position in a particular line of a poem. Although this approach is very important in that it attempts to address the problem of poetry generation by formalizing the syntactic features characterizing poetry it suffered from the need to record explicitly all the rules and predicates about the data.

Manurung [11] proposed a poetry generation system, which, given some metrical constraints as input, it uses the dynamic programming technique of chart generation to efficiently construct all valid paraphrases of a natural language utterance. The chart could be used both as a transducer for the production of logical form of utterance strings and as a generator from logical forms to strings [11]. The above technique addresses the issue of semantic meaning and paraphrasing of utterances via the use of a lexicon whose semantics subsume the semantics of input. With the advent of the Semantic Web tools and in particular with the introduction of ontologies as a tool enabling the representation of semantic relations between concepts, it is now possible to enrich the semantics of the poems generated via the use of a wider range of semantic relations between the concepts involved.

Another interesting technique was advocated in [12]. The basic strategy adopted in this case, was to produce poetry in collaboration with the user. The task is accomplished by firstly parsing the input line in order to analyze its poetic structure and then generating a new line. The output line relied on syllabification engine and a Support Vector Machine (SVM).

Creative text does not conform to the normal production rules governing non-poetic natural language text, creating an even more challenging problem. Manurung [13] refers to two distinct aspects of poetry generation that make it a unique and difficult problem in NLP [13]:

1. The interdependent linguistic phenomena and surface constrains due to 'unity' of poetry. Unity in this context refers to the fact that every single linguistic decision potentially determines the success of the poem.

2. Lack of clear, well-defined communicative goal

Although poetic text does not adhere to a valid definition, it satisfies the following properties:

1. Meaningfulness: The text must convey some conceptual message that is meaningful under some interpretation (this property actually holds for all types of text)

2. Grammaticality: A Poem must obey linguistic conventions that are prescribed by a given grammar and lexicon. Although this property also holds for all types of text, grammaticality in poetry is probably less constrained than that of ordinary texts and is governed by figurative language tropes.

3. Poeticness: A poem must exhibit poetic features such as phonetic patterns, rhythmic patterns and rhyme.

The above properties suggest that the semantic associations and tools used in poetry, impose further challenges to the syntactic analysis and semantic representation of poetic phrases. Our work focuses on the semantic aspects of a small subset of poetic tools in order to gain a better understanding of their nature and derive associations that can help in the representation of more complex phrases. The first two properties refer to all types of natural language text. However, the extent to which the properties apply in poetic writings may differ. For example, as stated in [13], "although this property (grammaticality) also holds for all types of text, grammaticality in poetry is probably less constrained than that of ordinary texts and is governed by figurative language 
tropes". Figurative language tropes (as stated in [14], constitute "a particular type of figures of speech that play with and shift the expected and literal meaning of words".

\subsection{Fuzzy domain relevant work}

One approach to the classification of textual tokens is via the use of fuzzy modelling of the concepts of the domain. Fuzzy modelling gained its interest due its successful applications in the industry for the solution of complex problems that cannot be resolved with precise mathematical formulations or logic due to their uncertain and ambiguous nature. Examples of areas in which it has been used successfully, are control systems, pattern recognition, expert systems, etc. The importance of fuzzy modelling lies to the fact that it tries to emulate human-like reasoning. The fundamental concept underpinning fuzzy modelling and logic, is the fuzzy set. As stated in [32] in conventional set theory, sets of real objects, are equivalent to, and, isomorphically described by, a unique membership function. However, the crisp boundaries of the above definition do not lend themselves easily to real applications since they do not take into account the inherent uncertainty and ambiguity of real life phenomena. In natural language, linguistic entities are characterized by ambiguity and therefore strict mathematical formulations are not applicable. Considering a universe of objects X, "a fuzzy set is a function $m: X \rightarrow[0,1]$ when, and only when, it matches some intuitively plausible semantic description of imprecise properties" [32]. This definition allows an object to belong to more than one fuzzy set. Consequently, it is particularly useful to the natural language domain and in particular to the classification and evaluation of similarity of textual entities, due to the fact that in this domain, there is no single and complete knowledge of the hierarchical relations between classes of words, neither of strict and precise definitions of classes, in order to draw inferences. Fuzzy inferences are drawn via fuzzy rules which are conditioned on fuzzy sets. Obviously, our identified set of rules (discussed in the next section) that lead to the identification of figures of speech are not strict inference rules.

Fuzzy modelling, where input datasets are textual resources, is cited in literature for the solution of IR and e-Learning problems. These works, are concerned primarily with the generation of fuzzy ontologies, usually by extending the traditional formal concept analysis and by employing fuzzy similarity measures to establish object-membership and hierarchical relations between concepts. Also, recent works in the engineering domain [30,31] provide further insight on fuzzy divergence and similarity among objects, which has not been addressed before [30].

The FOGA framework for the fuzzy ontology extraction [33] builds on Formal Concept Analysis (FCA) and fuzzy logic. The proposed framework consists of a fuzzy formal analysis component, a fuzzy conceptual clustering component, and a fuzzy ontology generation component. The Fuzzy Formal Concept Analysis (FFCA) component extends FCA so that membership to concepts is represented as a membership value in a fuzzy formal context [33]. In order to avoid the classification of similar objects into different classes due to small deviations in attribute values, formal concepts of the fuzzy concept lattice produced by FFCA are clustered by the Fuzzy Conceptual Clustering component. Finally, the Fuzzy Ontology generation component produces the fuzzy ontology using the concept hierarchy created by fuzzy conceptual clustering. This is an important framework for fuzzy inference, whose focus is the fuzzy classification of concepts. Our focus in this paper is on the ontological analysis of the conflicts between the assertions made by the literature devices under consideration and a real domain Human characteristics ontology. Textual data is inherently ambiguous in nature and fuzzy modelling proved to be a viable way of quantifying ambiguity.

In [29], lexical patterns leading to concept identification and extraction are determined by making use of POS-tagging. Then, statistical measures are used to determine the importance of words, and co-occurrences. The subsumption relations between concepts are computed according to a 
fuzzy relation membership function. Once the concepts and subsumption relations between concepts are established, a taxonomy of fuzzy domain concepts is extracted via the implementation of a fuzzy ontology construction algorithm.

In [34] an unsupervised approach is proposed to address a document classification problem which, taking advantage of the existence of readymade ontologies from many different domains, it assigns to each document a fuzzy mapping degree in the interval $[0,1]$. Each document considered, is mapped to each domain ontology with a certain degree of membership. The mapping degree of each document to each domain is then determined via fuzzy sets. This is an important work showing how to use existing domain ontologies to evaluate similarity with documents without any training data. It is however conditioned on the existence of relevant domain ontologies.

An important contribution regarding the assignment of fuzzy similarity relations between objects is cited in [30] and is concerned with the determination of the physical position, orientation and size of an electrically charged ellipsoidal conductor subjected to an external electric field. Crudely, the electrostatic potential of a given configuration of ellipsoidal conductor is represented and an $m \times m$ image $I$ to which, for each pixel, a grey level $y(i)$ is associated. The determination of membership of each $y(i)$ to $I$ is established via fuzzy sets. The method achieves comparable results to well-known fuzzy clustering techniques.

The works mentioned in the above paragraphs share the characteristics of a fuzzy domain and although their focus is on the fuzzy similarity and classification of objects in a fuzzy domain, their results relating to the extraction of semantically related concepts from textual resources are relevant to our work.

\section{THE USE OF WORDNET IN THE RECOGNITION OF FIGURES OF SPEECH}

Our data consists of poems extracted from the NLTK [15] Gutenberg file of William Blake and the Complete works of Shakespeare. Information about poems was extracted via natural language methods (e.g. regular expressions, parsing, etc.) and was inserted into a python dictionary for further processing so that, for example, the entry poems_dictionary $[k][j]$ holds information about the $\mathrm{j}^{\text {th }}$ line of the $\mathrm{k}^{\text {th }}$ poem. The dictionary also includes information about the noun phrases, prepositional phrases etc. Prepositional phrases were extracted via the use of the Pattern [16] library for Python and grammar patterns. Using WordNet [6] and some basic rules (for example the three following rules stated in Python) about the categories of words, we have been able to derive a list of phrases adhering to the definition of figures of speech.

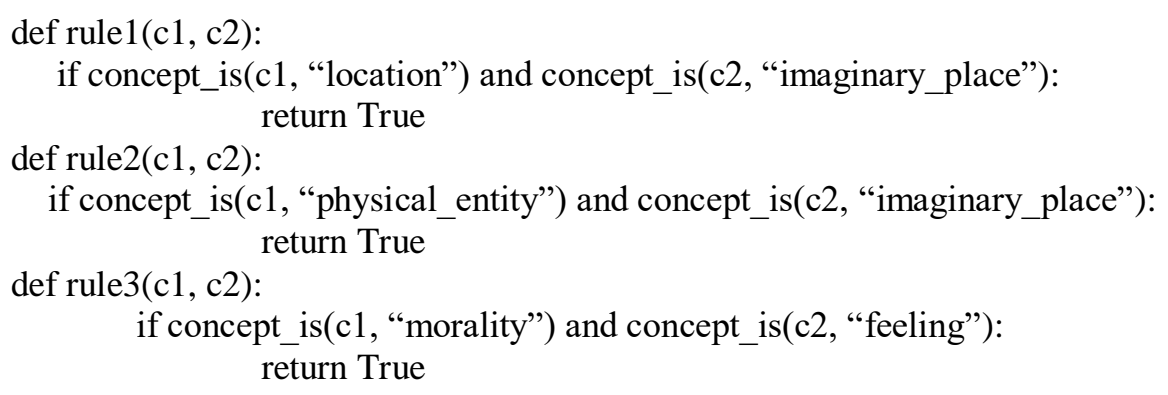

In the table below we include more examples of rule instances presented as ordered pairs of concepts with examples of figures of speech derived by these rules. 
International Journal of Artificial Intelligence and Applications (IJAIA), Vol.11, No.1, January 2020

Table 1. Examples of figures of speech derived from a set of conflict-identification rules

\begin{tabular}{|l|l|l|}
\hline Concept1 & Concept2 & Example \\
\hline offspring & abstraction & The daughter of beauty \\
\hline morality & feeling & Virtue of Love \\
\hline physical_entity & imaginary_place & Sword of Heaven \\
\hline artifact & feeling & beams of love \\
\hline furnishing & body_part & curtain of flesh \\
\hline physical_entity & spiritual_being & vales of har \\
\hline
\end{tabular}

Our analysis above, has encouraged us to develop an algorithm in order to extract those pairs of hypernyms that determine the type of phrase (i.e. poetic non-poetic), represented as a relation between noun concepts, $c 1$ and $c 2$. Let us denote the set of all relations as Relations. The steps followed in the algorithm, are outlined in the following subsection, including relevant definitions as appropriate, for the clarification of the method used.

\subsection{Derivation of poetic vs. non-poetic figures of speech}

Firstly, we define the associated to relation for pairs of hypernyms, as follows:

Definition 2 ( relation Assoc) $\left(\mathrm{h}_{\mathrm{i}}, \mathrm{h}_{\mathrm{j}}\right)$ is associated to $\mathrm{r}$ where $\mathrm{r}=(\mathrm{c} 1, \mathrm{c} 2) \in$ Relations, denoted as: $\operatorname{Assoc}\left(\left(\mathrm{h}_{\mathrm{i}}, \mathrm{h}_{\mathrm{j}}\right), \mathrm{r}\right)$ iff exists $\mathrm{p} 1 \in$ hypernym paths of $\mathrm{c} 1$ st. $\mathrm{h}_{\mathrm{i}} \in \mathrm{p} 1$ and exists $\mathrm{p} 2 \in$ hypernym paths of $\mathrm{c} 2 \mathrm{st} . \mathrm{h}_{\mathrm{j}} \in \mathrm{p} 2$.

Informally, the term hypernym paths refers to a set of paths where each path is a list of synonyms ordered via a taxonomic is-a relation from a lexical entity to its root. In compliance with WordNet, we will use the function hypernym_paths $(l)$ in the future, to obtain the set of paths for a lexical entity $l$. Now we can define the set of all pairs of hypernyms where each pair is associated to a relation in Relations:

- $\mathrm{S}_{\text {all }}=\left\{\left(\mathrm{h}_{\mathrm{i}}, \mathrm{h}_{\mathrm{j}}\right): \operatorname{Assoc}\left(\left(\mathrm{h}_{\mathrm{i}}, \mathrm{h}_{\mathrm{j}}\right), \mathrm{r}\right)\right.$ for all $\mathrm{r} \in$ Relations $\}$

Then, we obtain the following sets:

- $\mathrm{S}_{-\mathrm{np}}=\left\{\left(\mathrm{h}_{\mathrm{i}}, \mathrm{h}_{\mathrm{j}}\right)\right.$ in $\mathrm{S}_{\text {all }}$ where $(\mathrm{c} 1, \mathrm{c} 2)$ in Non_Poetic_Relations $\}$

- $\mathrm{S}_{\mathrm{p}}=\left\{\left(\mathrm{h}_{\mathrm{i}}, \mathrm{h}_{\mathrm{j}}\right)\right.$ in $\mathrm{S}_{\text {all }}$ where $(\mathrm{c} 1, \mathrm{c} 2)$ in Poetic_Relations $\}$

- $\mathrm{S}_{\mathrm{c}}=\left\{\left(\mathrm{h}_{\mathrm{i}}, \mathrm{h}_{\mathrm{j}}\right)\right.$ in $\mathrm{S}_{\text {all }}$ where $(\mathrm{c} 1, \mathrm{c} 2)$ in Poetic_Relations and $(\mathrm{c} 1, \mathrm{c} 2)$ in Non_Poetic_Relations $\}$

where Relations $=$ Poetic_Relations $\cup$ Non_Poetic_Relations

From the above sets, we can now derive sets of pairs of hypernyms that are only related to a specific type of relations and not others:

- $S_{-p}^{\prime}=\left\{\left(h_{i}, h_{j}\right)\right.$ in $H \times H:\left(h_{i}, h_{j}\right)$ in $S_{-p}$ and $\left(h_{i}, h_{j}\right)$ not in $\left.S_{-n p}\right\}$

- $S_{-}^{\prime}{ }_{-n p}=\left\{\left\{\left(h_{i}, h_{j}\right)\right.\right.$ in $H x H:\left(h_{i}, h_{j}\right)$ in $S_{-n p}\left(h_{i}, h_{j}\right)$ not in $\left.S_{\_p}\right\}$

where $\mathrm{H}$ denotes the sets of all hypernyms.

Now, since $S_{c}$ contains pairs of hypernyms that are related to both poetic and non-poetic relations, it consists mainly from less specific hypernyms at the higher levels of hypernym hierarchies. We eliminate those hypernym pairs in $S_{c}$ whose more specific hypernyms in the hypernym hierarchy 
are already associated to either the poetic or the non-poetic relations. Firstly, let us define the relation:

Definition 3 (relation higher_than) higher_than: $(\mathrm{H} \times \mathrm{H}) \times(\mathrm{H} \times \mathrm{H}){ }^{\circledR}$ Boolean such that for any two pairs of hypernyms $\left(\mathrm{h}_{\mathrm{i}}, \mathrm{h}_{\mathrm{j}}\right)$, and $\left(\mathrm{h}_{\mathrm{k}}, \mathrm{h}_{\mathrm{m}}\right)$ : higher_than $\left(\left(\mathrm{h}_{\mathrm{i}}, \mathrm{h}_{\mathrm{j}}\right),\left(\mathrm{h}_{\mathrm{k}}, \mathrm{h}_{\mathrm{m}}\right)\right)=$ True if exists $\mathrm{p}$ in hypernym_paths $\left(\mathrm{h}_{\mathrm{i}}\right) \mathrm{st}$. $\mathrm{h}_{\mathrm{k}}$ is a superordinate of $\mathrm{h}_{\mathrm{i}}$ and exists $\mathrm{p}$ in hypernym_paths $\left(\mathrm{h}_{\mathrm{j}}\right) \mathrm{st} \mathrm{h}_{\mathrm{m}}$ is a superordinate of $h_{j}$.

Then,

- $\mathrm{S}_{\mathrm{c}}:=\mathrm{S}_{\mathrm{c}}-\left\{\left(\mathrm{h}_{\mathrm{k}}, \mathrm{h}_{\mathrm{m}}\right)\right.$ : exists $\left(\mathrm{h}_{\mathrm{i}}, \mathrm{h}_{\mathrm{j}}\right)$ in $\mathrm{S}_{-\mathrm{p}}^{\prime}$ st. higher_than $\left.\left(\left(\mathrm{h}_{\mathrm{i}}, \mathrm{h}_{\mathrm{j}}\right),\left(\mathrm{h}_{\mathrm{k}}, \mathrm{h}_{\mathrm{m}}\right)\right)\right\}$

- $\left\{\left(\mathrm{h}_{\mathrm{k}}, \mathrm{h}_{\mathrm{m}}\right)\right.$ : exists $\left(\mathrm{h}_{\mathrm{i}}, \mathrm{h}_{\mathrm{j}}\right)$ in $\mathrm{S}_{-}{ }_{\mathrm{n} p} \mathrm{st}$. higher_than $\left.\left(\left(\mathrm{h}_{\mathrm{i}}, \mathrm{h}_{\mathrm{j}}\right),\left(\mathrm{h}_{\mathrm{k}}, \mathrm{h}_{\mathrm{m}}\right)\right)\right\}$

We can then choose among the pairs of hypernyms in sets $\mathrm{S}_{-}^{\prime}$, $\mathrm{S}_{-}{ }_{-n p}$ those pairs that do not have any superordinates in the same set.

- $\mathrm{S}^{\prime \prime}{ }_{\mathrm{p}}=\left(\mathrm{h}_{\mathrm{i}}, \mathrm{h}_{\mathrm{j}}\right)$ in $\mathrm{S}_{{ }_{\mathrm{p}}} \mathrm{p}$ : not exists $\left(\mathrm{h}_{\mathrm{k}}, \mathrm{h}_{\mathrm{m}}\right)$ in $\mathrm{S}_{-\mathrm{p}} \mathrm{s}$ st. higher_than $\left.\left(\left(\mathrm{h}_{\mathrm{i}}, \mathrm{h}_{\mathrm{j}}\right),\left(\mathrm{h}_{\mathrm{k}}, \mathrm{h}_{\mathrm{m}}\right)\right)\right\}$

- $\mathrm{S}_{\text {_np }}=\left\{\left(\mathrm{h}_{\mathrm{i}}, \mathrm{h}_{\mathrm{j}}\right)\right.$ in $\mathrm{S}_{-}{ }_{\mathrm{n} p}$ : not exists $\left(\mathrm{h}_{\mathrm{k}}, \mathrm{h}_{\mathrm{m}}\right)$ in $\mathrm{S}_{-}{ }_{\mathrm{np}} \mathrm{st}$. higher_than $\left.\left.\left(\mathrm{h}_{\mathrm{i}}, \mathrm{h}_{\mathrm{j}}\right),\left(\mathrm{h}_{\mathrm{k}}, \mathrm{h}_{\mathrm{m}}\right)\right)\right\}$

The importance of this restriction is that it gets the pairs of hypernyms as high as possible in their respective hierarchies and avoids redundancy. In this way we have managed to extract many more hypernym pairs relating to the classes of relations.

The results obtained via the use of the above approach are promising as shown in table 2 below. We realize that the relations not classified as either poetic or non-poetic are partly due to further processing required on our data at the preparation stage including the recognition of entities and numbers.

Table 2. Summary of results

\begin{tabular}{|l|l|l|l|}
\hline & Poetic & Non-poetic & Total \\
\hline Categorized as poetic & 206 & 0 & $\mathbf{2 0 6}$ \\
\hline Categorized as non-poetic & 0 & 4240 & $\mathbf{4 2 4 0}$ \\
\hline Other & 22 & 1302 & $\mathbf{1 3 2 4}$ \\
\hline Total & $\mathbf{2 2 8}$ & $\mathbf{5 5 4 2}$ & $\mathbf{5 7 7 0}$ \\
\hline
\end{tabular}

\subsection{Partially defined Ontology}

An initial, partially defined ontology is also derived as shown in Figure 1 below, aiming to provide a further ontological analysis of the associations between concepts used in texts (including poetic texts) which can be built manually and augmented via mappings with the hypernym hierarchies. We don't assume one universal ontology, and partiality in this sense is more realistic. This part of the ontology together with hierarchical information derived from WordNet hypernym hierarchies help to identify semantic associations of concepts in figures of speech. Let us consider, for example, the class Human_Gesture which includes the class Facial_expressions which in turn includes the object Smile. Assuming that only humans can make facial expressions in real life, we enforce the constraint that the domain of the relevant property: has_facial_expression is Human. Then, every assertion stating that an entity belonging to a different class has this property, will lead to a conflicting KB when added to the ontology.

The importance of a separate ontology is that hypernym hierarchies do not model important concept relationships other than hierarchical. Hypernyms do not provide information about disjointness relations between classes, and it cannot be inferred how, or, whether different entities 
are related. For example, how "tent" is related to Human, since WordNet [6] defines tent as a "structure that provides privacy and protection from danger". Having said that, we recognise that to record all possible relationships between concepts explicitly would not be feasible. However, abstracting away enables us to capture a wide range of relations using fewer classes.

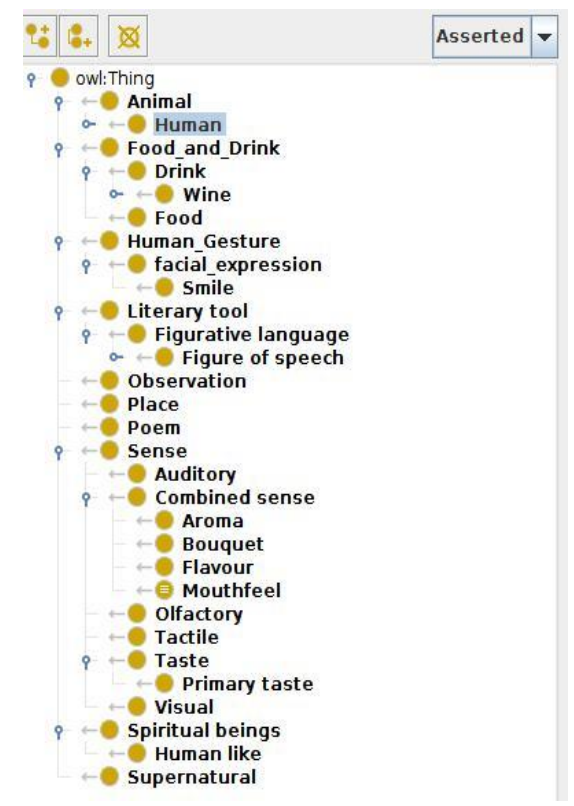

Figure 1. First draft of an ontology of humanly possessed qualities, and disjoint classes.

To motivate our discussion further, let us consider the prepositional noun phrase: 'smiles of heaven'. If 'Heaven' is a member of the class 'Supernatural' in $\mathrm{O}_{\mathrm{R}}$, which is disjoint with class 'Human', and the domain of property has_facial_expression is 'Human', then asserting that 'Heaven' has property has_facial_expression will end up in a conflicting KB. Nonetheless, this inconsistency (please refer to the notion of inconsistency below) leads to the identification of a personification phrase. Although it is not within the scope of this paper to provide a final solution to the problem of inconsistency, we discuss possible ways of addressing inconsistency in the following subsections either through reification or contextualized ontologies. But, in order to do that we first need to define the Syntax and Semantics of terminological knowledge bases.

\subsection{Basic Syntax and Semantics of Terminological Knowledge Bases}

DL based formalisms, like OWL DL, are 'a family of class-based knowledge representation formalisms equipped with well-defined model-theoretic semantics' [17]. In order to discuss conflicts with ontological knowledge we firstly need to refer to the definition of an ontology, and the notions of interpretation and satisfiability.

\subsubsection{Ontology}

An ontology in this paper is described as a structure $<\mathrm{A}, \mathrm{T}>$ where $\mathrm{T}$ denotes a DL TBox (a set of terminological axioms) and A denotes a DL ABox (a set of grounded assertions). An interpretation $\mathrm{I}$ of an ontology $\mathrm{O} \equiv<\mathrm{A}, \mathrm{T}>$ consists of a domain $\Delta^{\mathrm{I}}$ and an interpretation function (') such that the relations in Table 2 are satisfied. Note that the axioms referring to the domain and range of properties take their usual meaning and are neglected due to limitation of space. 
Table 2. Syntax and Semantics of Basic DL.

\begin{tabular}{|c|c|c|}
\hline Constructor Name & Syntax & Semantics \\
\hline $\begin{array}{l}\text { atomic concept } \mathrm{A} \\
\text { abstract role } \mathrm{R} \\
\text { individuals I } \\
\text { top concept } \\
\text { bottom concept }\end{array}$ & $\begin{array}{c}\mathrm{A} \\
\mathrm{R} \\
\mathrm{O} \\
\backslash \text { top } \\
\perp\end{array}$ & $\begin{array}{l}\mathrm{A}^{\mathrm{I}} \subseteq \Delta^{\mathrm{I}} \\
\mathrm{R}^{\mathrm{I}} \subseteq \Delta^{\mathrm{I}} \times \Delta^{\mathrm{I}} \\
\mathrm{o}^{\mathrm{I}} \in \Delta^{\mathrm{I}} \\
\Delta^{\mathrm{I}} \\
\varnothing\end{array}$ \\
\hline $\begin{array}{l}\text { conjunction } \\
\text { disjunction } \\
\text { negation } \\
\text { exists restriction } \\
\text { value restriction }\end{array}$ & $\begin{array}{l}\mathrm{C}_{1} \sqcap \mathrm{C}_{2} \\
\mathrm{C}_{1} \sqcup \mathrm{C}_{2} \\
\neg \mathrm{C} \\
\exists \mathrm{R} . \mathrm{C} \\
\forall \mathrm{R} . \mathrm{C}\end{array}$ & $\begin{array}{l}\mathrm{C}_{1}{ }^{\mathrm{I}} \cap \mathrm{C}_{2}{ }^{\mathrm{I}} \\
\mathrm{C}_{1}{ }^{\mathrm{I}} \cup \mathrm{C}_{2}{ }^{\mathrm{I}} \\
\Delta^{\mathrm{I}}-\mathrm{C}^{\mathrm{I}} \\
\left\{\mathrm{x} \mid \exists \mathrm{y},(\mathrm{x}, \mathrm{y}) \in \mathrm{R}^{\mathrm{I}} \Rightarrow \mathrm{y} \in \mathrm{C}^{\mathrm{I}}\right\} \\
\left\{\mathrm{x} \mid \forall \mathrm{y},(\mathrm{x}, \mathrm{y}) \in \mathrm{R}^{\mathrm{I}} \Rightarrow \mathrm{y} \in \mathrm{C}^{\mathrm{I}}\right\}\end{array}$ \\
\hline Axiom Name & Syntax & Semantics \\
\hline concept inclusion & $\mathrm{C}_{1} \sqsubseteq \mathrm{C}_{2}$ & $\mathrm{C}_{1}{ }^{\mathrm{I}} \subseteq \mathrm{C}_{2}{ }^{\mathrm{I}}$ \\
\hline concept assertion & $\mathrm{C}(\mathrm{a})$ & $\mathrm{a}^{\mathrm{I}} \in \mathrm{C}^{\mathrm{I}}$ \\
\hline role assertion & $\mathrm{R}(\mathrm{a}, \mathrm{b})$ & $\left(a^{I}, b^{I}\right) \in R^{I}$ \\
\hline
\end{tabular}

The notion of satisfiability is closely related to the notion of consistency. A named concept $C$ in an ontology $O$ is satisfiable if and only if there is an interpretation $I$ such that $\mathrm{C}^{\mathrm{I}} \neq \varnothing$. The notion of satisfiability allows us to define inconsistency as follows:

Definition 4 (Inconsistency) An ontology is inconsistent if and only if it has no interpretation. Examples of inconsistent formulas (where the usual meanings of disjointness and domain apply) are:

- $\{\mathrm{A}(\mathrm{b}), \mathrm{A} \sqsubseteq \mathrm{B}, \mathrm{A} \sqsubseteq \neg \mathrm{B}\}$

- $\{\mathrm{C}(\mathrm{a}), \neg \mathrm{C}(\mathrm{a})\}$

- $\{\mathrm{A}(\mathrm{a}), \mathrm{B}(\mathrm{a}), \operatorname{Disjoint}(\mathrm{A}, \mathrm{B})\}$

- $\{$ Domain(R, A), Disjoint(A, B), R(a,b), B(a)\}

\section{Practical considerations about Consistency handling and REPRESENTATION}

As stated already, the figures of speech cannot be added as assertions in the A-Box of an ontology like $\mathrm{O}_{\mathrm{R}}$ since they will lead to inconsistency. In this sub-section we discuss how the existing tools and formalisms can be used to represent figures of speech. Although we are currently talking about the simplest form of figure of speech, the approaches considered may be extended to handle more complex phrases.

\subsection{Reification}

One way to overcome the problem of in consistency, is to represent figures of speech separately, as a particular class of (non-factual) statements, about which information is kept. This leads to the idea of reification, which is supported by the RDF syntax. RDF [18] supported the reification of statements via a special vocabulary [19] in order to represent information about triples. An example of a reified figure of speech is included below:

_ex1: rdf:type rdf:Statement;

rdf:subject \#Heaven;

rdf:predicate \#has_facial_expression;

rdf:object \#Smile; 
Information about_ex1 can be added as follows:

_ex1: exuri:has_author \#Whiltman;

_ex1: exuri:appears_in \#PoemId

In RDF, the subject of reification is intended to refer to a concrete realization of an RDF triple, such as a document or surface syntax, rather than a triple considered as an abstract object [20]. Other forms of reification include $\mathrm{N}$-ary relations [21], singleton properties [22], and Named Graphs [23]. Each of these approaches aims to solve a different problem. For example, N-ary relations [21] enable more than two individuals to participate in $\mathrm{N}$-ary relations [21], Named Graphs [23] enable the addition of provenance and trust information to web resources, and Singleton Properties [22] enable the creation of properties for a single statement. Different N-ary relation patterns are discussed in [21]. Each relation pattern uses a class to represent a relationship and $\mathrm{n}$ new properties to represent the association of each participating entity to the relation. This approach enables the addition of information about the entities participating to the relationship that a triple cannot express on its own. However, most of the above methods suffer from maintenance problems and the increased complexity caused by the number of new constructs created. Further, the extent to which contextualization needs to be formalized depends on the reasoning capabilities needed for the representation of figures of speech. An attempt to formalize contextualization of resources is provided in a separate subsection below.

\subsection{Contextualizing Ontologies}

This section relies heavily on the work done in contextualized ontologies in [25] in order to derive the basic conceptualization of a context-based representation of our domain. Before proceeding further, we need to redefine Ontologies taking into consideration context.

A contextualized representation of ontologies adopts the principles of locality and compatibility underpinning the local model semantics [26]. The principle of locality states that reasoning requires only part of what is potentially available and the principle of compatibility states that there is compatibility among the kinds of reasoning performed in different contexts [26]. In a context-based ontology approach, each ontology is indexed e.g. by an index $i$ and an ontology $\mathrm{O}_{i}$ defines a language $\mathrm{L}_{i}$. Every expression that appears either with an index $i$ or no index is assumed to be in the language defined by $\mathrm{O}_{i}$.

Definition 5 Let $I$ be a set of indices, $L$ be the disjoint union of $\mathrm{C}, \mathrm{R}$, and $\mathrm{O}$, the set of strings denoting concepts, roles and individuals, respectively. An OWL ontology with index $i$ is a pair $\left\langle i, \mathrm{O}_{i}\right\rangle$ where $i \in \mathrm{I}$ and $\mathrm{O}_{i}=\left\langle\mathrm{T}_{i}, \mathrm{~A}_{i}\right\rangle$ where $\mathrm{T}$ and $\mathrm{A}$ are a T-box and A-box respectively in [25]. Since we are modelling a domain consisting of conflicting ontologies, then the space of ontologies needs to be appropriately modelled, taking into consideration compatibility issues. Informally an OWL space is a set of ontologies appropriately indexed. Following [25], an OWL space is a family of ontologies $\left\langle i, \mathrm{O}_{i}>_{\mathrm{i}} \in_{\mathrm{I}}\right.$ such that every $\mathrm{O}_{i}$ is an ontology, and for each $i \neq \mathrm{j}$, the $j$-foreign language of $\mathrm{O}_{i}$ is contained in the local language of $\mathrm{O}_{j}$.

Definition 6 A context space is a pair: $\left\langle\left\{\left\langle i, \mathrm{O}_{i}\right\rangle\right\}_{\mathrm{i} \in \mathrm{I}},\left\{\mathrm{M}_{\mathrm{ij}}\right\}_{\mathrm{ij} \in \mathrm{I}}>\right.$ where $\left\{\left\langle i, \mathrm{O}_{i}\right\rangle\right\}_{\mathrm{i} \in \mathrm{I}}$ is an OWL space (a set of indexed Ontologies) and $\left\{\mathrm{M}_{i j}\right\}_{i j} \in_{\mathrm{I}}$ is a family of mappings from $i$ to $j$ for all pairs $i$, $j \in \mathrm{I}$.

Before we define our context space, let us define an interpretation for an owl space as stated in [25] and a new relation < between indexed ontologies:

Definition 7 (OWL localized interpretation [25]) An OWL interpretation with local domains for the OWL space $\left\langle i, \mathrm{O}_{i}\right\rangle_{i \in \mathrm{I}}$, is a family $\mathrm{I}=\left\{\mathrm{I}_{i}\right\}_{i \in \mathrm{I}}$, where each $\mathrm{I}_{i}=\left\langle\Delta^{\mathrm{I}}\right.$, (.)Ii $\rangle$, called the local interpretation of $\mathrm{O}_{i}$ is an interpretation of $\mathrm{L}_{i}$. 
The above definition deviates slightly from the definition of OWL interpretation with local domains in [25] since it does not include holes [25]. Instead, we assume a subsumption relation between the properties of the ontologies of the owl space assuming a top ontology whose properties subsume the properties of the other ontologies in the owl space.

Definition 8 (relation $<_{\mathrm{r}}$ ) For any two indexed ontologies $\left\langle p, \mathrm{O}_{p}\right\rangle$, and $\left\langle t, \mathrm{O}_{t}\right\rangle$, let $\mathrm{I}=\left\{\mathrm{I}_{p}, \mathrm{I}_{t}\right\}$ be an OWL interpretation with the usual properties:

1. $(p: C)^{I_{t}}=C^{I_{p}} \cap \Delta^{I_{t}}$

2. $(p: \mathrm{r})^{I_{t}}=r^{I_{p}} \cap \Delta^{I_{t}} \times \Delta^{I_{t}}$

3. $(p: a)^{I_{t}}=a^{I_{t}}$

Then, the relation $\mathrm{O}_{\mathrm{p}}<_{\mathrm{r}} \mathrm{O}_{\mathrm{t}}$ holds between two indexed ontologies $\left\langle p, \mathrm{O}_{p}>\right.$ and $\left\langle t, \mathrm{O}_{t}\right\rangle$ if and only if $(p: r)^{I_{t}} \subseteq(t: r)^{I_{t}}$

Let us define our OWL space as the set of indexed ontologies: $\left\{\left\langle\right.\right.$ poetic, $\left.\mathrm{O}_{\text {poetic }}\right\rangle,\langle$ partial, $\mathrm{O}_{\text {partial }}>$ \}, <top, $\mathrm{O}_{\text {top }}>$, where $\mathrm{O}_{\text {poetic }}<{ }_{\mathrm{r}} \mathrm{O}_{\text {top }}$ and $\mathrm{O}_{\text {partial }}<{ }_{\mathrm{r}} \mathrm{O}_{\text {top }}$. Notably, the OWL space may contain more indexed ontologies. In our example, we assume that $\mathrm{O}_{\text {partial }}$ has mappings that relate its concepts to WordNet hypernyms. Assume that the following axioms hold for the OWL space just defined:

1. poetic: Smile $\sqsupseteq$ poetic: Facial_Expression

2. poetic: Supernatural(poetic: "heaven")

3. poetic: Smile(poetic: "bigsmile")

4. poetic: hasFacialExpression(poetic: "heaven", poetic: "bigsmile")

5. partial: Human_being ᄃ $\neg$ partial: Supernatural

6. $\forall$ partial: hasFacialExpression.Smile $ᄃ$ partial: Human_being

7. poetic: Human_being $\equiv$ partial: Human_being

8. poetic: Smile $\equiv$ partial: Smile

9. poetic: Supernatural $\equiv$ partial: Supernatural

10. poetic: hasFacialExpression $\subseteq$ top: hasFacialExpression

11. partial: hasFacialExpression $\subseteq$ top: hasFacialExpression

In the above OWL space, we assume that the domains and the interpretations of objects are the same in all ontologies apart from the extensions of properties. If we let $\mathrm{I}=\left\{\mathrm{I}_{\text {poetic }}, \mathrm{I}_{\text {partial }}, \mathrm{I}_{\text {top }}\right\}$ to be the set of local interpretations of ontologies $\mathrm{O}_{\text {poetic }}, \mathrm{O}_{\text {partial }}, \mathrm{O}_{\text {top }}$ respectively, then it follows that $\mathrm{I}_{\text {top }} \vDash$ hasFacialExpression (poetic: "heaven", poetic: "bigsmile"). At the same time, $\mathrm{I}_{\text {top }} \vDash$ $\neg$ partial: hasFacialExpression(poetic: "heaven", poetic: "bigsmile".

\subsection{Programming tools}

RDFLib enables the creation of separate graphs and contexts in a Dataset using the RDFLib [24], which can be very useful although, being an RDF triple based approach, it does not enable OWL reasoning in the sense of contextualized ontologies discussed in the previous subsection. The RDFLib package [24] enabled us to create named graphs and contexts, serialised and queried as RDF triples sharing the same URI. Datasets in RDFLib can be queried using SPARQL. It can also be used to create and store hierarchical relations, which in our case were mapped to Hypernym hierarchies. Ontologies were created and modified semi-automatically to reflect the OWL primitives with hypernym entities mapped to the partial ontology.

\section{CONCLUSION AND FUTURE WORK}

The current paper sets the foundations for the identification and semantic analysis of concepts included in figures of speech by following a semi-manual ontological approach. With the use of a set of a few basic rules concerning WordNet classes of entities and terminological knowledge 
derived from a real world ontology concerning the constraints on properties, we have been able to identify a set of prepositional noun phrases constituting figures of speech. The results, were encouraging suggesting that some poetic phrases used as literature tools can be recognized and analyzed due to their contradictory nature when compared to a real domain ontology. Based on these results we then suggested a method for the automatic categorization of poetic vs non-poetic phrases of our domain by collecting the most relevant hypernyms associated with each of the concepts involved. The semantic analysis gave us an in-depth understanding of the various features of poetic vs non-poetic statements. In particular, the use of an ontology describing human features can help (via the use of constraints, e.g. disjointness, domain and range) to identify conflicting assertions made by personification phrases and can be mapped to hypernym classes. Automating this task is currently under consideration. More complex phrases will be investigated in the future as well as a refinement of ontological relationships and concepts used, in order to aid the task of classification of phrases in parallel with an automated approach for the automatic extraction of particular figures of speech.

The paper also reviewed some of the eminent fuzzy domain approaches to the generation of fuzzy ontologies and the implementation of fuzzy measures of similarity. Our domain shares the conditions of uncertainty and ambiguity of these approaches. Finally, we have considered the challenges involved in the representation of figures of speech due to the inconsistency caused when added to the A-Box of a real-domain ontology such as the one employed in order to conceptualize observations regarding Human behavior and properties. We have referred to different approaches and have considered the application of contextualized ontologies for the solution of the inconsistency problem.

\section{REFERENCES}

[1] Literary Devices Editors, (2013) "Figure of Speech" [Online]. Available: https://literarydevices.net/figure-of-speech/

[2] Poetry.org. (2005) "what is poetry" [Online]. Available: http://www.poetry.org/whatis.htm.

[3] Hugo Concalo Oliveira. (2009) "Automatic generation of poetry: an overview", CISUC, DEI, University of Coimbra, Tech. Rep. 1.

[4] Abrams, M.H and Geoffrey G. Harpham. (1999) "A Glossary of Literal terms, Boston", MA: Thomson, Wadsworth.

[5] Project Gutenberg. Retrieved February 21, 2016, [Online]. Available: www.gutenberg.org.

[6] Miller, George A. (1995) "WordNet: A Lexical Database for English", Communications of the ACM, Vol. 38, No. 11.

[7] Literary Devices Editors (2013) "Personification" [Online]. Available: https://literarydevices.net/personification.

[8] Blake, W., and Blake, W. (1992) "Songs of innocence; and songs of experience". New York: Dover.

[9] Bailey, R.W. (1974) "Computer Assisted Poetry: The writing machine is for everybody", Computers in Humanities, Cambridge University Press, pp283-295.

[10] Toivanen, J.M., Toivonen, H. and Valitutti, A. (2013) "Harnessing constraint Programming for poetry composition", In Proceedings of the Fourth International Conference on Computational Creativity, pp160-167.

[11] Manurung, H.M. (1999) "Chart Generation of Rythm-Patterned Text", Proceedings of the First International Workshop on Literature in Cognition and Computers.

[12] Amitava Das and Bjorn Gamback. (2014) "Poetic Machine: Computational Creativity for Automatic Poetry Generation in Bengali", ICCC.

[13] Manurung, H. (2004) "An evolutionary algorithm approach to poetry generation", PhD thesis, University of Edinburgh.

[14] LitCharts Editors (2015) "Literary devices and terms" [Online]. Available: https://www.litcharts.com/literary-devices-and-terms.

[15] Loper, Edward and Bird, Steven (2002) "NLTK: The Natural Language Toolkit", Proceedings of the ACL-20 Workshop on Effective Tools and Methodologies for Teaching Natural Language Processing and Computational Linguistics, Association of Computational Linguistics, ETMTNLP, Vol. 1, pp63-70. 
[16] De Smedt, T. and Daelemans, W. (2012) "Pattern for Python", Journal of Machine Learning Research, Vol. 13, Issue 1, pp2063-2067.

[17] Flouris, G., Huang, Z., Pan, J., Plexoudakis, D. and Wache, H. (2006) "Inconsistencies, Negations and Changes in Ontologies", Proceedings of AAAI Conference on Artificial Intelligence.

[18] Hayes, Patrick and Patel-Schneider Peter Editors (2014) "RDF 1.1 Semantics" [Online]. Available: https://www.w3.org/TR/rdf11-mt.

[19] Brickley, Dan and Guha, R.V. (2004) "RDF Vocabulary Description Language 1.0:RDF Schema" [Online]. Available: https://www.w3.org/TR/2004/REC-rdf-schema-20040210.

[20] Wood, D., Lanthaler, M and Cyganiak, R. (2014) "RDF 1.1 Concepts and Abstract Syntax" [Online]. Available: https://www.w3.org/TR/2014/REC-rdf11-concepts-20140225.

[21] Hayes, P. and Welty, C. (2006) "Defining N-ary Relations on the Semantic Web" [Online]. Available: https://www.w3.org/TR/swbp-n-aryRelations.

[22] Nguyen, V., Bodenraider, O. and Sheth, A. (2014) "Don't like RDF Reification? Making Statements about Statements Using Singleton Property, Proceedings of the International World Wide Web Conference, pp759-770.

[23] Carrol, J., Bizer, C., Hayes, P. and Stickler, P. (2005) "Named Graphs, Provenance and Trust", Proceedings of the 14th International Conference on World Wide Web, pp613-622.

[24] Boettiger, C. (2018) "rdflib: A high level wrapper around the redland package for common rdf publications, Zenodo Publisher.

[25] Bouquet, P., Giunchiglia, F., Harmelen, F., Serafini, L. and Stuckenschmidt, H. (2003) "C-OWL: Contextualizing Ontologies", Proceedings of the second International Semantic Web Conference, Lecture Notes in Artificial Intelligence, No. 2870, pp164-179.

[26] Ghidini, C. and Giunchiglia, F. (2001) "Local Model Semantics, or Contextual Reasoning = Locality + Compatibility", Journal of Artificial Intelligence, Vol. 127, No. 2, pp221-259.

[27] Leite, M.A., and Ricarte, I.L. (2008) "Using Multiple Related Ontologies in a Fuzzy Information Retrieval Model" WONTO.

[28] Attia, Z.E., Gadallah, A.M., and Hefny, H.A. (2014) "Semantic Information Retrieval Model: Fuzzy Ontology Approach".

[29] Lau, R.Y., Song, D., Li, Y., Cheung, C., and Hao, J. (2009) "Toward a Fuzzy Domain Ontology Extraction Method for Adaptive e-Learning”, IEEE Transactions on Knowledge and Data Engineering, Vol. 21, pp800-813.

[30] Versaci, M., Foresta F.L., Morabito F.C. and Angiulli, G. (2018) “A fuzzy divergence approach for solving electrostatic identification problems for NDT applications”, International Journal of Applied Electromagnetics and Mechanics. Vol. 57. pp1-14.

[31] Cacciola, M., Calcagno, S., Megali, G., Pellicanò, D., Versaci, M., and Morabito, F.C. (2010) "Wavelet Coherence and Fuzzy Subtractive Clustering for Defect Classification in Aeronautic CFRP”, International Conference on Complex, Intelligent and Software Intensive Systems, pp101107.

[32] Bezdek, J.C. (1993) "Fuzzy models-What are they, and why?" [Editorial], IEEE Transactions on Fuzzy Systems, Vol. 1.

[33] Quan, T.T Hui, S.C and Cao, T.H. (2004) "FOGA: A Fuzzy Ontology Generation Framework for Scholarly Semantic Web".

[34] Arab, A.M, Gadallah, A.M., and Salah, A. (2017) "Training-less Multi-Domain Classification Approach using Ontology and Fuzzy sets", International Journal of Computer Science and Information Security. Vol 15. No.1. pp282-288.

[35] Panayiotou, C. (2019), "An Ontological Approach to the Extraction of Figures of Speech", The 5th International Conference on Artificial Intelligence and Applications, Dubai.

\section{AUTHOR}

Professor at the Cyprus University of Dr. Christiana Panayiotou is an Assistant Technology.

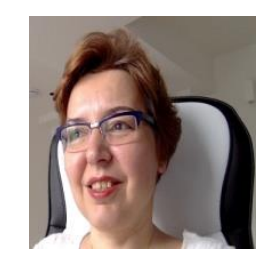

